Hewitr, L. F. (1954). J. gen. Microbiol. 11, 261-271.

\title{
Autoadaptation of Bacterial Viruses and its Effect on Bacterial Variation and Evolution
}

\author{
By L. F. HEWITT \\ Serum Research Institute, Medical Research Council, Carshalton, Surrey
}

SUMMARY: The adaptation of viruses to attack new hosts which originally are resistant greatly widens the sphere of influence of viruses on bacterial variation and evolution. The viruses attacking diphtheria bacilli are highly specific in their host range activity but many of them are readily adapted to lyse strains of other serological types. The range of adaptability of viruses is largely unknown and studies on the specificity and adaptability of bacterial viruses are complicated by the phenomenon of autoadaptability. Although virus-carrying bacterial strains are normally resistant to the viruses with which they are symbiotically infected it is now found that in many cases the viruses can be readily adapted to attack and lyse the parent bacterial cells. This has been found to occur spontaneously in cultures, and accounts for many cases of bacterial variation and dissociation. The existence of closed evolutionary chains in lysogenic bacterial strains provides the explanation of many phenomena and difficulties in studies of phage typing and specificity. A further effect of significance in this field is the possibility of obtaining hybrid viruses by genetic recombination between an externally infecting virus and a virus already symbiotically infecting a bacterial cell. These effects can be elicited experimentally but they have also been observed to occur spontaneously in stock cultures and, it is suggested, may account for processes of bacterial variation and evolution controlled by viruses.

It has been shown that bacterial viruses may constitute an important factor in controlling bacterial evolution (Hewitt, 1952, 1953) supplementing the normal biological processes of natural selection and the survival of the fittest. Bacterial viruses are of very wide occurrence, and among many bacterial species a high proportion of strains are symbiotically infected with viruses, but these are frequently highly specific in their range of activity. Despite the common occurrence of bacterial viruses therefore the chance of any bacterial strain encountering a specifically active virus would be remote were it not for the phenomenon of adaptation by which viruses can be trained to attack originally resistant bacteria. It should be emphasized that the term adaptation is used in its widest sense without prejudice to the mechanism of the process which may well be by means of mutation and selection.

This communication is concerned with some of the factors involved in this adaptation process which are largely unknown but are of importance in practical problems of bacteriology and phage typing as well as in more speculative aspects.

\section{MATERIALS AND METHODS}

Most of the experiments have been conducted with diphtheria cultures, both recently isolated and strains in laboratory culture which have been described previously (Hewitt, 1947). Both tryptic digest and papain digest broths have 
been used for the cultivation of bacteria and propagation of viruses. It was found necessary to test each batch for suitability for virus propagation before use. For the detection of lysis on solid media the incorporation of $10 \%$ horse serum as a growth adjuvant was found desirable. Special culture media for specific purposes are described in later sections.

In preliminary experiments the virus preparations used were the supernatant fluids from centrifuged cultures. Since diphtheria bacilli are readily killed by heating, sterilization of the preparations could be attained by heating at $50^{\circ}$ for $30 \mathrm{~min}$. The diphtheria phages are destroyed by heating at $55^{\circ}$ and for obtaining sterile unheated virus preparations filtration was used. There are heavy losses of virus in Pasteur Chamberland L3 candles and Seitz filter pads but filtration through sterile collodion membranes of average pore diameter $0.7 \mu$ was found satisfactory. General methods of virus propagation and testing have been described previously (Hewitt, 1952).

\section{RESULTS}

\section{Detection of lysogenicity}

In order to demonstrate that a bacterial strain is symbiotically infected with a virus it is necessary to have available a susceptible bacterial strain which will be lysed by any virus particles liberated by the bursting of an occasional lysogenic bacterial cell. Fisk (1942) placed drops of suspected lysogenic strains on smears of other bacterial strains on agar plates and examined for evidence of lysis. In the case of many diphtheria strains which yield rough irregular growth appearances on solid media traces of lysis are difficult to detect, and no clearing of broth cultures by viruses can be observed. It is often necessary therefore, to augment the potency of the virus preparation by attempting its propagation on the suspected sensitive strain before the possibility of lysogenicity can be excluded. In many lysogenic strains an occasional bacterial cell bursts, liberating virus particles, and the number of these can sometimes be increased by ultraviolet irradiation of the culture (Lwoff, Siminovitch \& Kjeldegaard, 1950). It is probably impossible to be certain that a strain is not lysogenic, but conversely, proof that bacteria are carrying a symbiotic virus can be quite definite.

The procedure adopted for detecting bacterial lysogenicity is as follows: the culture or supernatant fluid is first spotted on to a number of possibly sensitive strains on agar plates, then a similar test is applied to strains first subjected to ultraviolet irradiation, and finally the supernatant fluids or sterile filtrates from ultraviolet irradiated cultures are first propagated in broth cultures of the sensitive strain before testing for lytic activity on agar plates.

In a preliminary experiment 24 diphtheria strains were each added to cultures of 6 other strains selected for possible sensitivity. The supernatant fluids from each mixed culture were added to fresh subcultures of the 6 possibly susceptible strains and this serial propagation was continued several times. When the supernatant fluids were spotted on agar plate cultures 
of the 6 strains it was found that in the case of 20 out of the 24, strains some lytic activity had been produced against strain $\mathbf{H 1 0}$. This strain was therefore selected as a suitable indicator strain for the detection of lysogenesis and for the propagation of diphtheria phages. Similarly, Smith \& Jordan (1931) had selected an indicator diphtheria strain in their experiments which will be commented upon later.

Using $\mathrm{H10}$ as propagating strain a considerable number of fairly potent virus preparations have been obtained. The specificity of the phage preparations was quite narrow; they produced lysis only in $\mathrm{H} 10$ and a few closely related strains. It was found possible, however, to adapt the virus preparations to lyse strains of other serological types by several serial passages with them. Different strains appeared to vary in the ease with which viruses could be adapted to lyse them, but having been adapted to lyse one strain the preparation could then be re-adapted to lyse fresh strains and so on apparently indefinitely. In some cases the original specificity was immediately lost; in other cases the host specificity was only slightly modified in each adaptation and the ability to attack the original host was lost only after several readaptations.

The range over which the host specificity of viruses can be altered is not known, so attempts were made to adapt various staphylococcus phages to lyse diphtheria strains. None of the staphylococcus phages examined had any direct lytic effect on diphtheria strains, so passage of the supernatant culture fluids in fresh subcultures of the diphtheria strains was carried out as already described in the case of diphtheria phages. After a varying number of serial passages, varying from 2 to 18, a number of supernatant fluids obtained from staphylococcus phages added to the diphtheria indicator strain were found to possess definite lytic action on $\mathrm{H} 10$. When other diphtheria strains were examined it was found that lytic filtrates could be obtained in some cases but in others no lysis was observed. This unexpected result of obtaining a lytic agent for diphtheria bacilli by serial subculture with staphylococcus filtrates was subjected to careful examination in order to provide a reasonable explanation. The most obvious explanation of accidental contamination was eliminated by frequent repetitions of the experiment under different conditions. The staphylococcus phages had not however been quantitatively converted into diphtheria phages as shown by titrations in successive passages. The amount of diphtheria phage appearing was quite independent of the fall in staphylococcus phage titre which occurred by dilution in successive passages. Also antisera prepared against the original staphylococcus phage had no measurable effect on the diphtheria phage preparations. Experiments with antisera against diphtheria phages were unsatisfactory since sera of reasonable potency could not be obtained. The phages were not of high enough titre to be good antigens, and possibly the antigenic response of the rabbits had been impaired since they had to be protected against the toxin present in the diphtheria filtrates either passively with antitoxin or by preliminary active immunization with diphtheria toxoid.

Since neither chance contamination nor quantitative conversion of staphy- 
lococcus phage to diphtheria phage could account for the results a more complicated explanation appears necessary. There may, for example, be an interaction between the staphylococcal filtrates and possible lysogenic phages carried by the sensitive diphtheria strains, as found for example by Anderson \& Felix (1952) in the case of typhoid bacilli. More information was needed about the phage-host relationship in the case of Corynebacterium diphtheriae, about the adaptability of phages and about the possibility of genetic recombination.

Further experiments described in this and subsequent papers indicate that the diphtheria strains investigated are indeed lysogenic and capable of both adaptation and genetic recombination. In some cases the diphtheria strains were found to be carrying two or more distinct viruses and there seems little doubt that the development of lysins by subculturing staphylococcus filtrates with certain diphtheria strains is related to the presence of viruses carried by lysogenic strains.

\section{Autoadaptation}

In an experiment designed to lead to the isolation of a new series of viruses from 11 diphtheria strains, each one was subcultured with each of 10 strains making 110 cultures in all. After $18 \mathrm{hr}$. incubation the supernatant fluid from each of the mixed cultures was added to a fresh subculture of the propagating strain. The serial passages were continued several times, and then the supernatant fluids were tested for lytic activity on serum agar plates. In twenty-four cases some lysis was observed and the new viruses were isolated. Perhaps the most interesting observation, however, was that in three cases which had been included as controls the lytic activity observed was in cultures in which a strain had been subcultured with supernatant fluids obtained from previous serial subcultures of the same strain and no other bacterial strain had been added. It was found in fact that the symbiotic viruses carried by these strains had become adapted to lyse their own parent-carrying strains. Normally, of course, the host bacterium is resistant to lysis by its symbiotic virus, otherwise the host could not survive. This unexpected possibility of obtaining readily from a lysogenic strain a virus lysing the parent bacterium opens up new potentialities and explanations of experimental results.

In experiments previously mentioned lytic viruses had been obtained on a number of occasions by subculturing various strains with the indicator strain $\mathrm{H10}$. When this strain was subcultured with the supernatant fluid from previous subcultures of the same strain virus preparations were obtained after a few passages which readily lysed the parent strain. It is evident, therefore, that when the indicator strain $\mathrm{H} 10$ was subcultured with another strain and a virus attacking $\mathrm{H} 10$ was obtained it could not be assumed that the virus originated from the second insusceptible strain, since the virus could have arisen by adaptation from a symbiotic virus originally present in $\mathrm{H} 10$ itself. There were differences in the properties of viruses obtained by subculturing H 10 with different diphtheria strains. Some of these apparently had not originated from the indicator strain but from the strain incubated with it. As mentioned later, there is the further complication of the possibility of genetic recombination between the infecting and symbiotic viruses. 
The adaptation of a symbiotic virus to attack its own bacterial host has been repeated in a number of cases, but it may be difficult to demonstrate directly in the case of a lysogenic strain which liberates only a few virus particles spontaneously, and there may be too few particles present to include any mutants capable of the required adaptation. In these cases it is necessary first to propagate the lysogenic virus on a susceptible strain and, when a potent virus preparation has been obtained, to adapt the filtrate to the original parent strain. The disadvantage of this indirect procedure is that the susceptible bacterial strain used for the propagation may itself be lysogenic and liberate virus particles which intervene and complicate the autoadaptation process, rendering the origin of the resultant adapted virus preparation obscure. When it is possible to adapt a virus directly to attack its own parent bacterial strain the origin is indisputable.

\section{Spontaneous autoadaptation}

There is abundant evidence that autoadaptation of symbiotic viruses to attack their own hosts occurs spontaneously. For example, when old cultures of the strain $\mathrm{H} 10$ are freshly subcultured after a long interval the cultural appearance is sometimes abnormal and there is little growth in the second subculture. Spontaneous lysis has occurred and the virus susceptibility of the surviving cells is markedly subnormal. The explanation of these phenomena lies in spontaneous autoadaptation during storage of the old culture. Stored cultures are not in a state of completely suspended animation, and from time to time some of the lysogenic bacterial cells burst liberating free virus particles. An occasional mutant virus, perhaps less than one in a million of the virus particles present, is capable of adaptation to attack the parent bacterial strain. Once liberated these mutant viruses will multiply by infecting the host cells, whilst the normal virus particles are unable to proliferate since there is not a susceptible host present. In old stock cultures therefore there will be a gradual increase in the proportion of mutant viruses. On first subculturing aged stock cultures of bacterial strains of this type the mutant viruses multiply at the expense of the host cells and filtrates from these frequently have pronounced lytic activity against normal subcultures of the same strain. These mutant viruses are often of relatively feeble activity, they are readily superseded and tend to constitute only an almost undetectable minority in normal cultures, but their presence can be detected by special experimental methods as described in the next section.

\section{Inhomogeneity of cultures}

In a bacterial strain symbiotically infected with a virus (as indeed most strains are in the case of many bacterial species) the population of a culture containing hundreds of millions of cells is not completely homogeneous. Occasional cells contain virus particles differing from the remainder. Under ordinary cultural conditions this lack of homogeneity is not apparent since virus particles liberated from bursting cells infect any susceptible bacteria present and this has a homogenizing effect. As will be shown in a subsequent 
communication some viruses have a dominant effect and tend to overcome the effects of other less active ones under normal cultural conditions. If, however, this spread of virus infection through a culture is inhibited and individual members of the bacterial population are examined, the inhomogeneity can be detected. For successful attack on susceptible bacteria many viruses require the presence of divalent cations such as calcium (Bordet \& Renaux, 1928) and substances interfering with free calcium ions inhibit virus activity (Stassano \& de Beaufort, 1925). For this reason a number of diphtheria strains were subcultured in broth containing sodium oxalate so that virus multiplication was minimized, and then the subcultures were plated out on a solid medium on which a variety of colonial types can be distinguished readily. Heated blood agar plates containing tellurite can be used for this purpose, but much better differentiation is observed with laked blood agar plates containing $0.01 \%$ sodium selenite (Hewitt, 1948). On this medium a variety of colonies differing in size, shape and colour occur. Some organisms yield red colonies, others white and others with red and white bands. A typical experiment of this type was carried out with a gravis diphtheria strain $\mathbf{H} 874$. The strain was subcultured alternately in oxalate broth and on selenite blood agar plates, selecting colonies of different types. Most of the colonies were of medium size uniformly red in colour and when examined were found to be indistinguishable from the original parent cultures. Among these colonies were a few larger colonies, umbonate in shape, the central dome being bright red with a white peripheral annulus. These 'jam tart' colonies (H 874 JT) after several subcultures bred true and yielded a uniform colonial appearance when subcultures were plated out. The cultural characteristics were different from the original strain, growth in broth was poorer and less granular, producing a uniform turbidity. The original strain was virulent and lethal to guinea-pigs and in broth cultures toxin was formed, but the JT strain was completely avirulent and non-toxigenic, although starch was still fermented. The most interesting feature of the strain from the point of view of the present communication was that the virus it was carrying was different from that of the original strain. The parent strain was lysogenic and carried virus particles which attacked certain other strains, but the JT strain was symbiotically infected with a virus of different host range specificity which was capable of lysing the parent strain. It thus appeared to be a mutant virus which had arisen spontaneously from the virus particles carried by the parent strain. The bacterial cell carrying this mutant virus, when it divided, yielded daughter cells carrying the same mutant, but in normal broth cultures of the parent strain these would have been infected with the vigorous virus particles carried by most of the bacterial cells in the culture and the relatively feeble mutants would disappear. In the artificial conditions where virus multiplication and infection were inhibited by oxalate followed by segregation of the separate bacterial cells in the solid culture medium, it was possible for bacteria carrying mutant viruses to survive. When isolated in pure culture these aberrant strains survive and their characteristics have been maintained after many subcultures in normal broth. Some of these have been subcultured for 6 months without any apparent change or reversion. 


\section{Genetic recombination}

It has already been mentioned that the behaviour of a virus preparation may depend, not only on its origin, but also on the bacterial strains upon which it has been propagated. Some effects of this on virulence will be described in a subsequent communication but the host-range aspect should be considered here.

Some experiments with the three gravis diphtheria strains $\mathrm{H} 868, \mathrm{H} 873$ and $\mathbf{H ~ 8 7 4}$ are of interest in this connexion and are summarized in Table 1.

Table 1. Effect of propagating strain on host-specificity of viruses

\begin{tabular}{|c|c|}
\hline \multicolumn{2}{|c|}{ Virus preparation } \\
\hline $\begin{array}{l}\text { From } \\
\text { strain }\end{array}$ & $\begin{array}{c}\text { Propagating } \\
\text { strain }\end{array}$ \\
\hline H873 & H 868 \\
\hline H873 & H874 \\
\hline H874 & H868 \\
\hline H874 & H873 \\
\hline
\end{tabular}

\begin{tabular}{|c|c|c|}
\hline H868 & H873 & H 874 \\
\hline+ & 0 & + \\
\hline+ & 0 & + \\
\hline+ & + & 0 \\
\hline+ & + & + \\
\hline
\end{tabular}

Virus preparations obtained from strain $\mathrm{H} 874$ lyse the strains $\mathrm{H} 868$ and $\mathrm{H} 873$, whilst the virus from strain $\mathrm{H} 873$ lyses strains $\mathrm{H} 868$ and $\mathrm{H} 874$. When H874 viruses are propagated on strain $\mathrm{H} 868$ (giving virus 874/868) the filtrate lyses both $\mathrm{H} 868$ and $\mathrm{H873}$ but not the original parent strain $\mathrm{H} 874$. However, when $\mathbf{H 8 7 4}$ viruses are propagated on strain $\mathrm{H873}$ the resultant filtrate (874/873) lyses not only strain $\mathrm{H} 868$ and $\mathrm{H} 873$ but also $\mathrm{H874}$, the parent strain from which the virus preparation was originally obtained. Moreover, when strain $\mathrm{H868}$ is treated with the virus 874/873 the resistant culture which grows out is found to consist of organisms carrying a virus, which can be liberated by ultraviolet irradiation. The virus filtrate thus obtained is found still to be active against all three strains $\mathrm{H868}, \mathrm{H} 873$ and H 874. It would appear that genetic recombination had occurred between the infecting virus from strain $\mathbf{H 8 7 4}$ and the symbiotic virus carried by strain $\mathrm{H} 873$ and that this genetic recombination persisted when the virus was propagated on strain 868 , and also when it entered into symbiotic relationship with $\mathbf{H 8 6 8}$. The possibility has not been absolutely excluded that the virus preparation is a mixture of viruses and not a genetic recombinant. It is interesting to note that when the virus from $\mathrm{H} 873$ is propagated on strain $\mathrm{H} 874$ the resulting virus preparation still has the properties of $\mathrm{H} 873$ virus and is not a genetic recombinant. Regardless, however, of the final theoretical explanation of the phenomenon the practical point remains that the properties of $\mathbf{H 8 7 4}$ viruses depend upon the strains upon which they are propagated.

\section{DISCUSSION}

Among many species of bacteria, lysogenicity is very common and there is a growing volume of evidence that this widespread symbiotic infection with viruses plays a decisive role in controlling bacterial behaviour and evolution. 
In attempting to elucidate the detailed mechanism of the virus control of bacterial variation complications are encountered. Bacterial viruses can be propagated only in bacterial cells and it is becoming increasingly obvious that the host on which a virus is propagated can affect the properties of the propagated virus. The virus preparation obtained by propagation on a given strain may be profoundly different from the original virus used as the seed. It is well-known that passage of a virus through a new host may modify its host-specificity and this effect has been noted in the case of animal and plant viruses as well as bacterial viruses. The important point that it is wished to emphasize here, however, is that viruses can often be readily adapted to attack the parent bacterial hosts with which they have entered into a symbiotic relationship.

Normally a lysogenic strain is resistant to the attack of the virus with which it is symbiotically infected; otherwise virus particles liberated by an occasional bursting cell would immediately lyse the parent culture. It is now found, however, that symbiotic viruses can, in fact, be adapted to attack the parent strain. This autoadaptation is quite different from the lysis induction process studied by Lwoff (1953). It has been shown by Lwoff et al. (1950) that a lysogenic culture may be induced by ultraviolet irradiation or other treatment to lyse and liberate virus particles. The virus liberated, however, does not attack the parent strain and the treatment has merely induced the cells either to produce complete virus particles from previous intermediate forms (Lwoff, 1953) or, at any rate, so to modify the host-virus relationship that the host cell bursts and liberates free virus particles. Boyd (1951), in the cases he has studied, finds evidence of the existence of two kinds of virus mutants, one kind being lytic and the other, instead of causing susceptible cells to lyse, tends to form stable symbiotic infections of the bacteria attacked. In neither the induction phenomena studied by Lwoff nor the mutations of Boyd, however, is there change in host-range specificity and the liberated virus particles are not able to lyse bacterial cells carrying the same virus. For free virus particles to cause lysis of bacterial cells carrying the same virus their host specificity has first to be modified or adapted.

As already indicated, the term adaptation is used here, not in the narrow sense of a non-hereditary cytoplasmic alteration, but with the broad meaning indicated by Stanier (1953). Adaptation therefore is used to describe the totality of processes by which the specificity of a virus preparation is modified to enable it to multiply in a new host. This may well involve both genetic and environmental factors, including perhaps the selection of an occasional virus mutant capable of gaining entry into bacterial cells which resist the attack of most members of the virus population. Once the parent virus has gained entry the daughter virus particles produced by replication processes may be modified, both by the synthetic mechanisms of the host cell and by other virus particles or proviruses already present. The experiments described in this communication and other preliminary observations suggest that factors influencing virus specificity are very complex (Burnet \& Lush, 1936). Propagation of virus particles on different hosts may yield virus preparations of totally 
different properties and specificities, the factors involved including mutation, selection, genetic recombination with other viruses and environmental influences.

There are several practical consequences of this autoadaptation phenomenon. When two bacterial strains, $\mathbf{A}$ and $\mathbf{B}$, are cultured together and a virus preparation results which lyses one of them, say $A$, it is not safe to assume that it necessarily arises from the other strain, B, since it may arise by autoadaptation of the virus carried by $\mathbf{A}$ itself.

As envisaged previously (Hewitt, 1953) it is possible to have a completely closed evolutionary system consisting only of a lysogenic bacterial strain and the virus it carries. By autoadaptation of the virus to attack the host strain an evolutionary chain is initiated. The parent strain is attacked by the mutant virus and the resistant organisms only survive. In turn the virus may mutate to attack this resistant strain and so on, there being no limit to the evolutionary steps that can occur. This process has been simulated in the laboratory by adapting lysogenic viruses to attack the host strain, isolating the consequent resistant bacterial strain and re-adapting the virus to attack this new strain. Steps in the chain can, however, be recognized in cultures which have not been subjected to artificial interference. In ordinary bacterial cultures virus mutation occurs and bacterial variants resistant to these mutants arise but the bacterial population remains fairly homogeneous because the viruses present reinfect the bacterial cells and a given virus tends to become dominant and overwhelm other less vigorous rivals. It is possible, however, to inhibit this homogenization process by adjusting the culture medium to restrict virus multiplication and infection. One method which has been found successful is to rid the medium of calcium ions which are necessary for virus multiplication and then to plate out the culture on solid medium on which viruses cannot be transmitted from a bursting cell to other bacteria in the culture. By alternate subculture in calcium-deficient liquid media and on a differential solid medium it is a simple matter to select from ordinary bacterial cultures variant bacteria carrying viruses different from the main bulk of the bacterial population. These variant bacteria frequently differ in important biological properties and variations in virulence, metabolism, colonial form, morphology, etc., have been found. The viruses they carry vary and some may be found which attack the parent culture, but if these viruses are of a recessive character they will tend to disappear in ordinary cultures where dominant viruses overcome their effect. Changes in cultural conditions may, however, disturb the balance between dominant and recessive viruses and, for example, in old cultures a complete and spontaneous change in cultural characters is sometimes observed on subculture. The explanation of these apparently spontaneous variations remains obscure until the effect of bacterial viruses is taken into account.

The phenomenon of autoadaptation probably accounts for the results of Smith \& Jordan (1931) and Stone \& Hobby (1934) who observed lytic action on one diphtheria strain when it was subcultured with a high proportion of other strains. At times this strain showed a 'suicidal tendency' and died out. This 
strain showed marked variability, and it seems probable that it was lysogenic and the virus it carried was readily adapted to lyse the parent strain. In the case of staphylococci Fisk (1942) found a number of very variable strains which after storage liberated a virus which lysed cultures isolated when plating out the parent strains. Autoadaptation of the carrier virus would account for Fisk's conclusion that variation in stock cultures 'may be an indication of alteration in the normal relationship of phage to bacterium'. Oeding (1950) has described colonial and other variants in diphtheria cultures including 'an eroded bacteriophage-like appearance' in the colonies which may readily be explained on the basis of virus autoadaptation.

A further property of viruses may complicate the issue when more than one virus is present. Evidence has accumulated that sometimes when a lysogenic bacterial strain is attacked by a virus to which it is susceptible, the virus particles liberated when it bursts may differ from the infecting particles and may be hybrids produced by genetic recombination between the infecting virus and the symbiotic virus carried by the original strain. Virus preparations with the host specificity of the two strains are obtained consistently from some gravis diphtheria strains.

Hershey \& Rotman (1949) report genetic recombination between mutants of Escherichia coli $\mathrm{T}_{\mathbf{2}}$ bacteriophage simultaneously infecting bacterial cells. This result was confirmed by Visconti \& Delbruck (1953), and a similar result obtained with $T_{4}$ bacteriophage by Doermann \& Hill (1953). It is assumed that during multiplication of the virus in the cell there is random mating of the particles giving rise to the hybrid viruses. As far as is known there has, however, been no report of hybridization between virus particles attacking a cell from outside and virus particles already present in lysogenic cells. In the present experiments, however, when the virus $(a)$ from one strain (A) infected another diphtheria strain (B), which was already carrying a virus $(b)$, the virus preparation from the lysed cells lysed both strains $A$ and $B$ and behaved as if it were the hybrid $a b$ with the host specificities of both viruses. On the other hand, when virus $b$ was propagated on strain $\mathbf{A}$ the resulting virus preparation did not behave as the hybrid $a b$ but like the original virus $b$ with the admixture of relatively few particles of $a$ from the lysogenic cells. More work is required to establish definitely the existence of this recombination, since recent experiments indicate the presence in the two strains, not of two viruses $a$ and $b$ only but four $\left(a_{1}, a_{2}, b_{1}\right.$ and $\left.b_{2}\right)$. These complications provide the explanation of both the difficulties and value of phage typing methods. The intervention of lysogenic virus in the preparation of typing phages makes reproducible results difficult to obtain (Smith, 1948), but increases the possibility of differentiating strains of diverse origin (Craigie \& Yen, 1938; Williams \& Rippon, 1952).

\section{REFERENCES}

Anderson, E. S. \& Felix, A. (1952). Variation in Vi-phage II of Salmonella typhi. Nature, Lond. 170, 492.

Bordet, J. \& Renaux, E. (1928). L'autolyse microbienne transmissible ou le bactériophage. Ann. Inst. Pasteur, 42, 1283. 
Boyd, J. S. K. (1951). Observations on the relationship of symbiotic and lytic bacteriophage. J. Path. Bact. 62, 501.

Burnet, F. M. \& Lush, D. (1936). Induced lysogenicity and mutation of bacteriophage within lysogenic bacteria. Aust. J. exp. Biol. med. Sci. 14, 27.

Craigre, J. \& Yen, C. H. (1938). The demonstration of types of B. typhosus by means of preparations of type II Vi-phage. Canad. publ. Hlth J. 29, 448.

DoermanN, A. H. \& HiLl, M. B. (1953). Genetic structure of bacteriophage T4 as described by recombination studies of factors influencing plaque morphology. Genetics, 38, 79.

Fisk, R. T. (1942). Studies on staphylococci. I. Occurrence of bacteriophage carriers among strains of Staphylococcus aureus. J. infect. Dis. 71, 153.

Hershey, A. D. \& Rotman, R. (1949). Genetic recombination between host-range and plaque-type mutants of bacteriophage in single bacterial cells. Genetics, 34, 72.

Hewitr, L. F. (1947). Serological typing of C. diphtheriae. Brit. J. exp. Path. 28, 338.

HewitT, L. F. (1948). Virulence and toxigenicity of different serological types of C. diphtheriae. Brit. J. exp. Path. 29, 18.

HewitT, L. F. (1952). Diphtheria bacteriophages and their relation to the development of bacterial variants. J. gen. Microbiol. 7, 362.

HewitT, L. F. (1953). Influence of bacteriophage on bacterial variation and evolution. In Adaptation in Micro-organisms. Symp. Soc. gen. Microbiol. 3, 276.

Lwoff, A. (1953). Lysogeny. Bact. Rev. 17, 269.

Lwoff, A., Siminovitch, L. \& KJeldegaArd, N. (1950). Induction de la production de bactériophages chez une bactérie lysogène. Ann. Inst. Pasteur, 79, 815.

Oeding, P. (1950). Type transformation in the diphtheria bacillus. Acta path. microbiol. scand. 27, 16.

Smith, G. H. \& Jordan, E. F. (1931). Bacillus diphtheriae in its relationship to bacteriophage. J. Bact. 21, 75.

Smrth, H. W. (1948). Investigations on the typing of staphylococci by means of bacteriophage. I. The origin and nature of lysogenic strains. J. Hyg., Camb. 46, 74 .

Stanier, R. V. (1953). Adaptation, evolutionary and physiological: or Darwinism among the micro-organisms. In Adaptation in Micro-organisms. Symp. Soc. gen. Microbiol. 3, 1.

Stassano, H. \& Beaufort, A. D. De (1925). Action du citrate de soude sur le principe lytique transmissible. C.R. Soc. Biol., Paris, 93, 1380.

Stone, F. M. \& Hoвву, G. L. (1934). A coccoid form of C. diphtheriae susceptible to bacteriophage. J. Bact. 27, 403.

Visconti, N. \& Delbruck, M. (1953). The mechanism of genetic recombination in phage. Genetics, 38, 5.

Williams, R. E. O. \& Rippon, J. E. (1952). Bacteriophage typing of Staphylococcus aureus. J. Hyg., Camb. 50, 320. 